\title{
AN EPIDEMIC, SIMULATING TYPHOID, CAUSED BY A PARAGAERTNER ORGANISM *
}

\author{
GEORGE H. ROBIN SON \\ (From the Bacteriological Laboratory of Brown University and the Providence Health \\ Department, Prozidence, R. I.)
}

Epidemics of typhoid fever due to the drinking of sewage-polluted water show a marked similarity in the ratio of those affected with gastro-enteritis to the cases of clinical typhoid fever. Comparison of several epidemics of this nature indicate that $40-60$ percent of those exposed to infection contract gastro-enteritis and 2-8 percent develop clinical typhoid. In epidemics in which food or milk, infected by typhoid carriers or incipient cases of typhoid, are the vehicles of infection, the incidence of typhoid is much higher and the accompanying gastro-enteritis is generally absent. During the investigation of the following epidemic a study was made of the cases of enteritis as well as of those pronounced as typhoid.

In the fall of 1913 there occurred an epidemic simulating typhoid among the Rhode Island party attending the Perry Centennial at Put-In-Bay, Lake Erie. The itinerary of the party and the sanitary aspect of the epidemic are thoroughly discussed in De Valin's report ${ }^{1}$ and that of Swarts. ${ }^{2}$ The party, consisting of 422 members, left Providence September 8 and upon reaching Buffalo was quartered on two steamers, the Rochester and the Greyhound, 300 being accommodated on the former and 122 on the latter. The courses of both boats were practically the same and both parties attended the same functions. The delegation returned to Providence on September 14.

Reports from 235 members of the party on the Rochester showed 122 cases, or 52 percent, of gastro-enteritis. Of the total 300 on board 42 , or 14 percent, developed clinical typhoid, including 6 deaths. The party on the Greyhound yielded upon inquiry 12 mild and temporary attacks of diarrhea" such as any change of water or diet might cause. In many ways this epidemic resembled others of infected-water origin, except that the number of typhoid cases was larger than the average.

* Received for publication March 26, 1915.

1. Publ. Health Rep., 1913, 28, p. 2761.

2. Fiealth Bull. Rhode Island State Board of Health, 1914, 3, p. 1. 
All possible sources of infection were carefully investigated by Dr. C. V. Chapin of the Providence Health Department, Dr. G. T. Swarts of the State Board of Health, and Dr. de Valin of the U. S. Public Health Service. The most salient points of the inquiry were: On September 11 the Rochester pumped water for drinking purposes from Lake Erie at points "considered decidedly unsafe;" during the trip a cook had performed his duties while suffering from typhoid fever; an electrician on the Rochester was taken sick with typhoid August 16; a maid and a water-tender both left the boat on September 20 feeling unwell and were later diagnosed as cases of typhoid. The maid died without giving a positive agglutination test with typhoid bacilli.

A check on the manner of infection is supplied in the case of members of the Newport Artillery, one of the organizations taking passage on the Rochester. The 83 members of this company were previously warned against the dangers of drinking water and so drank sparingly. None of them was affected with enteritis, but twenty-two of them developed typhoid symptoms about two weeks after their return. Eliminating those who drank little water, we find at least 122 cases of enteritis, or 80 percent, of the 152 members of the party who did drink freely of the water.

\section{SYMPTOMS}

The most common symptoms were enteritis, diarthea, malaise, and fever. "Forty-three presented symptoms varying from a mild paratyphoid, to a perfect clinical, typhoid character." Four of the patients were admitted to the Rhode Island Hospital, extracts from whose records follow.

CASE 16.-Admitted Oct. 4. Diagnosis, typhoid fever with lobar pneumonia complication.

Patient began to complain of headache, abdominal distress, pain in back, and fever, September 25. Went to bed September 28 because he felt weak. Appetite poor, bowcls regular. No nose blecd. No cough. Stated that he had had a similar attack six years before. Malaria four years before.

Well developed and well nourished. Face flushed, lips cracked. Tongue thickly coated on dorsum, red and moist at tips and edges. On chest and abdomen are a few circumscribed papules varying from the size of a pin to half the size of a pea, which disappear on pressure. No râles in chest. No murmurs of heart. Leukocytes 14,000 , polymorphonuclears 81 percent. No agglutination of typhoid bacilli.

Oct. 6.-Irrational, tosses about in bed, gets up if not watched. Spleen not palpable. 
Oct. 7.-More delirious, temperature $103 \mathrm{~F}$.

Oct. 8.-Temperature $101 \mathrm{~F}$. yesterday, during the night rose to $104 \mathrm{~F}$; pulse 130. Abdomen soft. No blood in stools. Impaired resonance in right lower lobe of lung. Died.

CAse 21.-Admitted Oct. 8. Diagnosis, typhoid fever.

Patient had headache and diarthea with cramps, Sept. 14. This lasted four days after which he felt as well as ever except that he became tired easily. On Oct. 4, he went on a canoe trip and became thoroughly exhausted. Since then has had severe headache. No chills and no fever. Had typhoid fever with a relapse nine years ago.

No râles, no heart murmurs, no tenderness on abdomen, no glandular enlargement. Letkocytes 5,600 , polymorphonuclears 63 percent, mononuclears 33 .

Oct. 10.-Temperature reached highest point, $103.5 \mathrm{~F}$.

Oct. 15.-Many typhoid bacilli in blood culture.

Oct. 20.-Temperature normal.

Nov. 4.-Discharged.

Case F-Admitted Oct. 4. Diagnosis, typhoid fever.

Felt sick on return trip Sept. 13. Headache, and later in the day watery and frequent bowel movements and vomiting. Had profuse diarthea up to Oct. 4. Has cramp in abdomen before every bowel movement and pain in the middle of the back almost constantly. No blood in stools. Has lost about 8 pounds. No nose bleed. Bad taste, appetite poor. High fever.

No tenderness in abdomen. Spleen palpable. No rose spots. Leukocytes 6,400 , polymorphonuclears 70.5 percent, mononuclears 28 .

Oct. 7.-Only complaint is dizziness. Spleen greatly increased in size. Serum agglutinates typhoid bacilli.

Oct. 10.-General condition good. Fever declining.

Oct. 13.-Patient at times irrational and slightly delirious, Temperature again elevated. No sign of perforation or hemorrhage.

Oct. 16.-During last three days has been profoundly toxic. During last twenty-four hours heart has been failing. Died.

CASE 40.-Admitted Oct. 29. Diagnosis, typhoid fever.

Symptoms began Oct. 22 with headache and loss of appetite, slight cough, but no nose bleed; slight fever, no chills, no vomiting, no pain in abdomen or chest or joints or back, no night sweats; bowels moved frequently last four days. Headache and malaise. Took complete typhoid inoculation one year ago.

Abdomen has suggestive rose spots. No tenderness or rigidity or spasms. Lower end of spleen felt. Leukocytes 11,000. Serum agglutinates typhoid bacilli.

Nov, 2.-Blood culture negative. Comfortable. Diarrhea stopped.

Nov. 5.-Feeling well but temperature is again $103 \mathrm{~F}$. at evening.

Nov. 8.-Is beginning to feel listless and a little weak; no headache and no abdominal pain.

Nov. 11.-Temperature up. Weak.

Nov. 21-Temperature normal.

Dec. 11.-Uneventful recovery. Discharged. 


\section{AGGLUTINATION}

The first agglutination of typhoid bacilli by the serum of any of the party was obtained September 28. This patient, however, had received prophylactic treatment for typhoid a few months previously, but during the investigation of his case it became evident that there was an infection of some kind among the members of the party. The last specimen of blood from this epidemic was sent in November 9. During this time 39 specimens of blood were received at the city and state health laboratories from those residing in or near Providence who attended the Perry Centennial.

These yielded the following results:

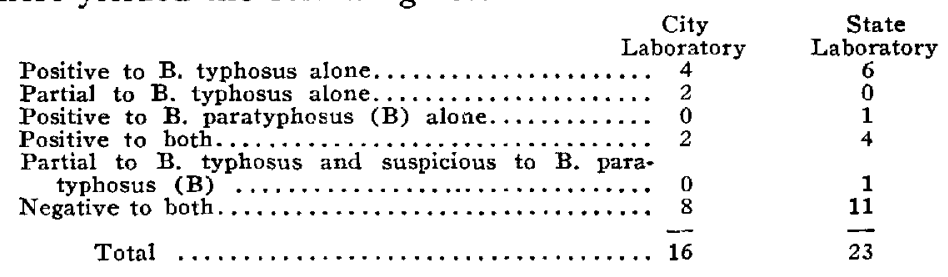

The large percentage of the cases which showed a reaction with $B$. paratyphosus (B) was noticeable throughout the epidemic.

\section{EXAMINATIONS OF FECES}

Specimens of feces were obtained from nineteen of the men in Providence. Through the efforts of Dr. C. V. Chapin specimens of feces and blood were obtained from the cook on the Rochester. About two months after the infection, through the kindness of Dr. Swarts, specimens were obtained from the convalescents in the Newport Hospital. A technic which had proven very successful was used in the examination of the feces. Small portions of the material were incubated in lactose peptone bile for six to twelve hours and then the bile culture was plated on Endo's medium. No typhoid organisms were isolated from any of the specimens. An organism was found in ten of the specimens, however, which resembled both typhoid and paratyphoid types in some respects, and differed in others.

This organism was obtained from feces of the cook of the Rochester and also from Cases 16 and 21.

It will be seen that the organism is similar to typhoid in that it does not ferment dulcite and does ferment dextrin. Like B. paratyphosus (B) it produces visible amounts of gas and ferments arabinose. 


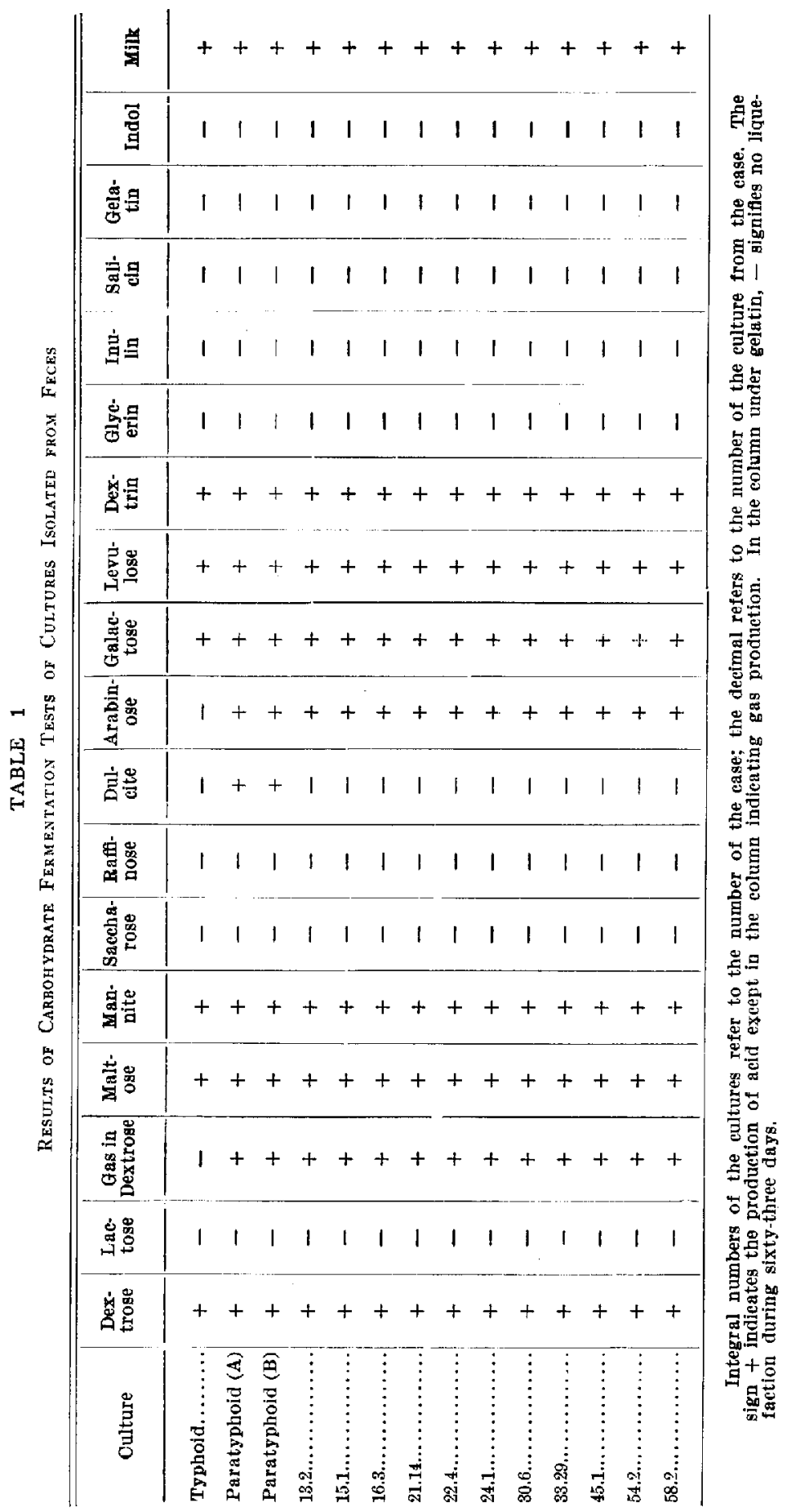


It is about the size of the typhoid bacillus, actively motile, and is gram-negative. According to its cultural characters and carbohydrate reactions, this organism would be classified as a member of the intermediate group. It could not be reckoned as a true gaertner organism on account of its production of permanent acidity in litmus milk. Evidently, it is one of the paragaertner organisms.

SERUm Reactions.-These cultures were tested with the serum of the patients from whom they were isolated, as well as with known typhoid and paratyphoid (A) and (B) sera. No reaction was obtained except in the case of the cook (Case 33). Cultures obtained from him agglutinated with his serum at a dilution of 1:60. Two rabbits were immunized against Culture 16.3 obtained from a fatal case. Five inoculations of $1 \mathrm{c} . c$. of killed twenty-four-hour broth cultures at intervals of 7 days in one rabbit and 5 days in the other failed to produce a serum reacting strongly with the organism injected or any of the other organisms isolated during the epidemic. One of the sera did produce an agglutination at a dilution of $1: 10$. Both sera, however, gave a positive reaction with $B$. typhosus at a dilution of $1: 60$. This would account for the positive agglutination tests among those of the party who were infected. The organism is evidently one which stimulates the formation of agglutinins slowly. Those patients who did not give positive agglutination tests had probably not been infected long enough.

Pathogenicity for Laboratory Animals.--The pathogenicity of the different strains evidently varied. Each strain was inoculated into a mouse but several of them did not succumb. Culture 16.3 isolated from one of the fatal cases seemed to be most pathogenic.

Mouse 2.-Weight, $20 \mathrm{gm}$. Inoculated subcutaneously with 0.2 c.c. twentyfour-hour broth culture of 16.3; death in 12-18 hours; congestion about point of inoculation, lungs somewhat congested. Organism isolated in pure culture from liver, lungs, heart, and kidneys.

Mouse 21 .-Weight, $25 \mathrm{gm}$. Inoculated subcutaneously with 0.25 c.c. twentyfour-hour broth culture of 16.3 ; evidently sick and feverish after forty-eight hours; diarrhea; died after fifty-five hours; lymph glands hemorrhagic, congestion at point of inoculation. Isolated organism from heart, liver, lung, stomach, and feces.

Mouse 12.-Jan. 20, fed bread soaked in twenty-four-hour broth culture of 16.3 ; Jan. 26 , fed bread soaked in twenty-four-hour broth culture 16.3 ; Jan. 30 , drank about 3 c.c. of twelve-hour-broth culture; Feb. 3, drank about 1 c.c. of twenty-four-hour broth culture; Feb. 6, looked sick; Feb. 7, died, eight days after drinking broth culttre. Axillary and lumbar lymph glands swollen and 
congested, gall-bladder very full, spleen apparently normal, diarrhea. Serum would not agglutinate culture. Organisms isolated from gall-bladder, small intestine, urinary bladder, and kidney. Heart and liver sterile.

Mouse 15.-Drank 2 drops twenty-four-hour broth culture of 16.3 ; died ten days later; appearance of organs normal. Organism isolated from heart and liver.

Guinea-pig 15.-Drank 2 c.c. twelve-hour broth culture of 16.3 ; died six days later; gall-bladder much enlarged, suprarenals enlarged, abundant peritoneal fluid, spleen normal, other organs apparently normal. Organism isolated from heart, liver, kidney, peritoneal fluid, gall-bladder, urinary bladder, and small intestine.

Guinea-pig 16.-Weight, $305 \mathrm{gm}$. Inoculated intraperitoneally, 1 c.c., twelvehour broth culture of 16.3; died four days later; abscess at point of inoculation, peritoneal cavity full of a white fluid, intestine and mesenteries inflamed, spleen apparently normal. Organism isolated from heart, liver, and peritoneal fluid.

Mouse 7.-Weight, $22 \mathrm{gm}$. Inoculated subcutaneously with $0.25 \mathrm{c.c}$, twentyfour-hour culture of 54.2 ; died after five days; congestion at point of inoculation; lymph glands swollen. Organism isolated from heart, liver, lungs, stomach, large intestine, and point of inoculation.

Mouse 14.-Drank 0.5 c.c. twenty-four-hour broth culture of 54.2 ; a week later refused to eat, evidently sick; died next day; appearance of organs normal. Organism isolated from stomach, small intestine, and large intestine. Heart, liver, and kidneys sterile.

Mouse 6.-Weight $22 \mathrm{gm}$. Inoculated subcutaneously with 0.25 c.c. twentyfour-hour culture of 33.29 ; died after fifteen days; spleen swollen; point of inoculation congested. Organism could be isolated only from point of inoculation.

Mouse 19.-Weight $20 \mathrm{gm}$. Inoculated subcutaneously with 0.2 c.c. twentyfour-hour broth culture of B. paratyphosus (B) ; died in forty hours; appearance of organs normal; lymph nodes congested; mesenteries congested. Organism isolated from heart, liver, kidneys, small intestine, and large intestine.

Examination of the organs in fatal cases showed no marked pathological changes. When infection occurs through the digestive tract, it is capable of producing enteritis and diarrhea in mice and guineapigs.

Mouse 12 was apparently unaffected ten days after eating solid food infected with culture of 16.3 but died on the eighth day after taking the organism in a liquid. Two other mice (Mice 13 and 15) died seven and ten days, respectively, after drinking liquid culture. Infection through the intestine is best accomplished by means of liquid rather than solid food.

PROPHYLACTIC TREATMENT OF MEMBERS OF THE PARTY

Sixteen members of the party had taken typhoid immunizing treatment less than a year before this epidemic. Of these, five were affected with enteritis and diarrhea and one (Case 40) was considered at the 
Typhold Epidemic Caused by Paragaertner Organism 455

hospital as having typhoid. Two other cases considered as typhoid fever (one of them Case 21) gave definite histories of having had it before. In view of the opinion among medical men that one attack of typhoid protects against another and that the prophylactic treatment protects for at least two years, these data would confirm our findings that it was not the typhoid bacillus with which the members of the party were infected.

\section{SUMMARY}

An epidemic has occurred resembling in many ways a water-borne typhoid epidemic. A careful bacteriological examination of feces of patients was negative for typhoid, but an organism was found which seemed to have the cultural characteristics of the paragaertner group. 